\title{
EDITORIAL
}

(อ) VIRAL HEPATITIS

\section{Viral hepatitis: progress and promise}

S ince January 2000 there have been more than 40,000 research articles published on the subject of viral hepatitis-basic, translational and clinical. Such investment has been well rewarded, with advances in our understanding of the mechanisms of disease and improved treatment options. As viral hepatitis remains a major global health problem, these advances are much needed. In recognition of the interest in this field, Nature Reviews Gastroenterology \& Hepatology commissioned a special focus issue to highlight some of the real-life issues pertinent to the present and future treatment and management of viral hepatitis.

As many individuals infected with $\mathrm{HBV}$ or $\mathrm{HCV}$ spontaneously clear the virus, treatment is traditionally reserved for those with chronic infection. Evidence has emerged, however, that treating an acute HCV infection can achieve a high success rate. Grebely, Matthews and Dore consider the epidemiology, diagnosis, natural history and treatment of acute HCV infection. As they explain, most new HCV infections in the developed world are acquired by injection drugs users, which brings its own unique set of challenges that culminate in a general reluctance to treat these patients. They argue that injection drugs users can be successfully treated, but that adherence is the key and that strategies to improve adherence are of paramount importance.

Progress in the treatment of hepatitis B means there are now seven approved medications. Kwon and Lok review the natural history of chronic $\mathrm{HBV}$ infection and treatment safety and efficacy, before answering some of the questions surrounding hepatitis B treatment. They stress the need to monitor treatment response and the importance of treatment adherence. For patients who do not respond to treatment, the management approach should be tailored according to treatment (current and past), type of failure, and also pretreatment patient and disease characteristics.

Treatment of decompensated liver disease related to HBV and HCV remains a challenge; however, as described by Fink and Jacobson, considering individuals on a case-by-case basis allows the options to extend beyond transplantation. They support the cautious use of IFN-based regimens in $\mathrm{HCV}$-infected patients. $\mathrm{HCV}$ should be eradicated in select patients prior to transplantation, provided they are monitored, as eradication can prevent post-transplantation recurrence. For $\mathrm{HBV}$-infected patients, treatment with antiviral agents is recommended and improvements in decompensation are possible in those who have a virologic response.

Therapeutic advances are set to continue in 2011, with the eagerly anticipated approval of the direct-acting antiviral agents (DAAs) telaprevir and boceprevir for chronic hepatitis $\mathrm{C}$, therapies that promise to deliver a new standard of care. Hofmann and Zeuzem summarize data from the phase III trials of telaprevir and boceprevir and consider how they will be introduced into clinical practice. They also discuss preliminary data on treatment with different DAA combinations, with or without IFN.

So what else is on the horizon for viral hepatitis? Response-guided treatment allows regimens to be more specifically tailored, taking factors such as viral genotype into consideration. Identifying host genotypes that confer treatment susceptibility or resistance (for example, IL28 polymorphisms that correlate with the response to IFN) takes things further and pretreatment genetic testing should ensure patients receive only beneficial treatments.

The identification of IL-32, a novel proinflammatory cytokine, and the potential role of microRNAs in viral replication are just two other discoveries that promise to enhance our understanding of the disease process. The quest to develop an effective vaccination against $\mathrm{HCV}$ continues with genetic engineering of adenovirus, while nanotechnology is being employed to help develop a potential diagnostic tool for widespread population screening. In addition, there are numerous HCV drugs in preclinical development and phase I and II trials, some of which are expected to be at least as efficacious as telaprevir and boceprevir, which suggests that in the future several different treatment options will be available.

Recent achievements in the field of viral hepatitis are certainly a cause for celebration, yet there is always more that can be done. Nonetheless, if research continues apace in the next 10 years, then there is every reason to be hopeful about what else might be accomplished.

doi:10.1038/nrgastro.2011.50

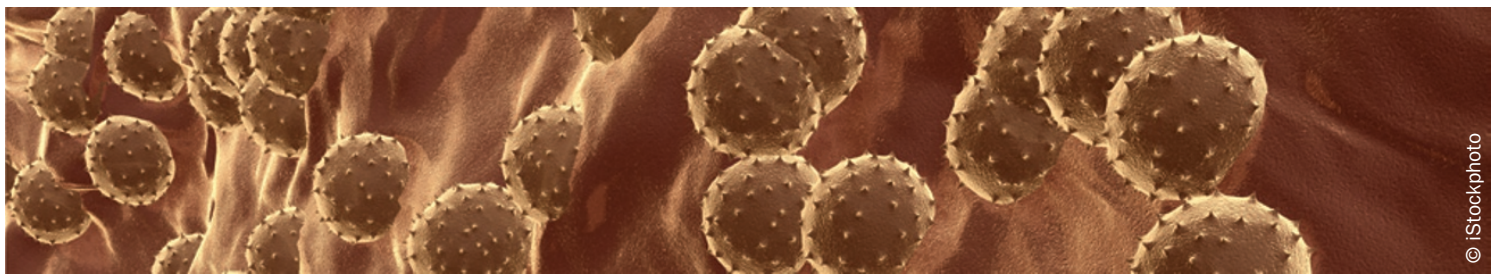

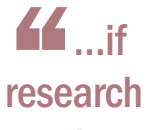

continues

apace ...

there is every reason to be hopeful... 77
Natalie J. Wood is the Chief Editor of Nature Reviews Gastroenterology $\&$ Hepatology.

Competing interests The author declares no competing interests. 